\title{
Verfassungsbeschwerde der Firma VisuKom gegen § 202c StGB
}

\begin{abstract}
Im Folgenden dokumentieren wir auszugsweise im Wortlaut die Erklärung, mit der sich Marco Di Filippo, der Geschäftsführer der VisuKom $\mathrm{GmbH}$, und ihr Anwalt Thomas Feil vor rund sechs Wochen an die Presse wandten. Sowohl die Beschreibung der kritischen Einsatzszenarien für sog. Hackertools als auch der wirtschaftlichen Konsequenzen des „Hackerparagraphen“ für das Unternehmen können hierbei stellvertretend für die gesamte Branche der IT-Security-Dienstleister stehen.
\end{abstract}

„Bamberg, 29. Oktober 2007 - In Deutschland wurde am 11.08.2007 der sog. ,Hackerparagraf' zur Bekämpfung der Computerkriminalität verkündet. Gemäß § 202c StGB zieht das ,Vorbereiten des Ausspähens und Abfangens von Daten' künftig eine Freiheitsstrafe von bis zu einem Jahr oder eine Geldstrafe nach sich. Marco Di Filippo, Geschäftsführer der VisuKom Deutschland $\mathrm{GmbH}$, hat nun in Kooperation mit Rechtsanwalt Thomas Feil eine Verfassungsbeschwerde (Aktenzeichen: 2 BVR 2233/07) gegen das in den Medien und der IT-Sicherheitsbranche stark umstrittene neue Strafrechtsänderungsgesetz eingereicht ...

Marco Di Filippo bietet als Geschäftsführer der Firma VisuKom Deutschland GmbH Dienstleistungen im Bereich ICT-Security (Information- and Communications Technology Security) an. Dabei werden u.a. auf ausdrücklichen Wunsch der Kunden so genannte Penetrationstests durchgeführt. Dies sind umfassende Sicherheitstests möglichst aller Systembestandteile und Anwendungen eines Netzwerk- oder Softwaresystems.

Dabei werden realitätsnahe Hackerangriffe simuliert, die es ermöglichen, Sicherheitsschwachstellen in den Netzinfrastrukturen von Unternehmen zu finden. Der Penetrationstest ermittelt somit die Empfindlichkeit des zu testenden Systems gegen derartige Angriffe. Das Anbieten von Dienstleistungen im Bereich ICT-Security, d. h. das Überprüfen von ICT-Netzinfrastrukturen auf Sicherheitsrisiken sowie das Aufspüren von Schwachstellen und deren Beseitigung, ist eine erlaubte Tätigkeit, die der IT-Sicherheit dient. Die Strafbarkeit der in § 202c StGB beschriebenen Handlungen verbietet dem Beschwerdeführer allerdings das Anbieten seiner bisherigen Dienstleistungen und stellt damit seine sowie die wirtschaftliche Existenz zahlreicher anderer Anbieter in diesem Umfeld in Frage.

,In der gesamten IT-Sicherheitsbranche besteht derzeit große Unsicherheit über die durch die Einführung des § 202c StGB geschaffene Möglichkeit der Strafverfolgung von grundlegenden Tätigkeiten ihres gesamten Gewerbezweigs', erklärt Marco Di Filippo ... Der für diesen Fall zuständige Rechtsanwalt Thomas Feil ... ergänzt: ,Die hier geführte Verfassungsbeschwerde ist von allgemeiner Bedeutung, da sie grundsätzlich verfassungsrechtliche Fragen aufwirft und die zu erwartende Entscheidung über den Einzelfall hinaus Sicherheit über die Rechtslage in einer Vielzahl gleich gelagerter Fälle schafft. Mit dem Ergebnis dieses Verfahrens kann für diesen Berufszweig endgültig Klarheit über die Strafbarkeit seiner Tätigkeit erzielt werden.'“

Dokumentation: Thomas Böcker 